\title{
Micro- to Nanoscale Morphologies and Chemical Components of Soils Investigated by SEM-EDS for Forensic Science
}

\author{
Yingzi Liu, ${ }^{1}$ Yumei Li, ${ }^{2}$ Qiang Li, ${ }^{2}$ Jie Bao, ${ }^{2}$ Dakui Hao, ${ }^{2}$ Zhiwen Zhao, ${ }^{1}$ Dongxue Song, \\ Jiajia Wang, and Zhiheng $\mathrm{Hu}^{2}$ \\ ${ }^{1}$ Department of Criminal Science and Technology, Shandong Police College, Jinan 250014, China \\ ${ }^{2}$ School of Biological Science and Technology, University of Jinan, Jinan 250022, China \\ Correspondence should be addressed to Qiang Li; chm_liq@ujn.edu.cn
}

Received 19 December 2014; Accepted 21 January 2015

Academic Editor: Qichen Wang

Copyright (C) 2015 Yingzi Liu et al. This is an open access article distributed under the Creative Commons Attribution License, which permits unrestricted use, distribution, and reproduction in any medium, provided the original work is properly cited.

As a kind of microscale physical evidence, soil can provide significant assistance to forensic science. In this study, soil samples that were collected from eighteen different regions of Shandong Province, China, were examined by scanning electron microscopeenergy dispersive spectrometer (SEM-EDS). The homogeneities and diameters of the samples were evaluated by SEM which has been applied to observe objects at nanoscale. The soil from Jiaxiang, a city in Eastern Shandong Province, showed the maximal particle diameter and the sample from Liaocheng, another Eastern city in Shandong Province, showed the best homogeneity. The mass fraction and molar percentages of nine inorganic elements in all samples were analyzed by EDS. Oxygen and silicon showed the highest content in all of these samples. However, different samples exhibited their own characteristic elements, which can help to discriminate them from other samples. In this regard, SEM-EDS-based homogeneity and element analysis might be used as a fast and reliable technique for the soil criminological analysis in Shandong Province.

\section{Introduction}

Soil provides the nutrients and water required by terrestrial plants growth and exchange energy. Soil is influenced by various biotic and biological factors, such as climate, early formation of texture, microbial environment, and human activities. Therefore, the soil from various regions has tremendous differences in the components of metal, minerals, and the microbial communities $[1,2]$.

There is a strong connection between the soil and people's lives. Soil on the body or belongings of a person could be used as trace evidence in a large amount of criminal cases. As a kind of trace evidence, soil has played an important role in forensic science [3-6].

The physical properties of soil, including $\mathrm{pH}$, color, density gradient, and inorganic components, are traditionally examined as evidences. With the development of modern technology, detection techniques for using soil as evidence are simultaneously improved [2]. Scanning electron microscopy is a type of electron microscope that produces image of a sample by scanning it with a focused beam of electrons. The electrons interact with electrons in the sample, producing various signals containing information about the sample's surface topography and composition. Energydispersive X-ray spectroscopy (EDS) is an analytical technique used for element analysis or chemical characterization of samples. SEM-EDS can determine the sizes, shapes, and elemental compositions of particles, which has been adopted by many researchers to identify different particles and link them to possible sources. Cengiz et al. collected soil samples from seventeen different regions of Istanbul city, and concluded that the SEM-EDS method can discriminate the soil evidences [7]. To the best of our knowledge, morphology characteristics and elemental composition analysis about the soil particles in Shandong Province, China, have never been reported.

A biological method, T-RFLP analysis of Shandong's soil microbial community, was previously reported for forensic science [8]. As a supplement to the T-RFLP analysis, the SEMEDS analysis technique was used as an abiotic method to 

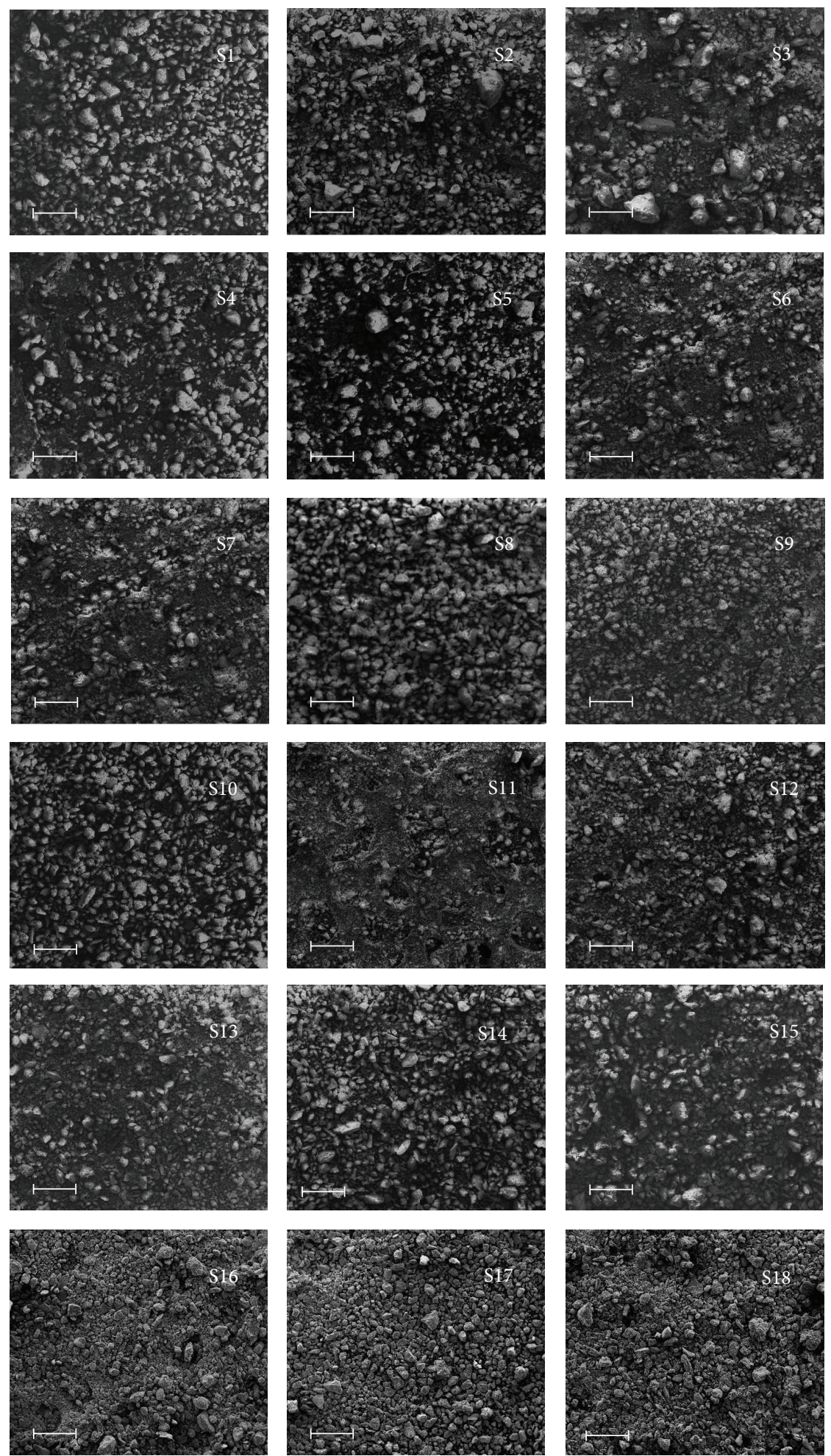

FIGURE 1: SEM images of soil from eighteen regions in Shandong province. S1 to S18, represent Zaozhuang, Yantai, Penglai (Yantai), Wulian (Rizhao), Changyi (Weifang), Rongcheng (Weihai), South mountainous area (Jinan), Liaocheng, Laiwu, Jiaozhou (Qingdao), Jiaxiang (Jining), Heze, Kenli (Dongying), Xiajin (Dezhou), Zouping (Binzhou), Qufu, Taian, and Zibo, respectively. The scale bar is $200 \mu \mathrm{m}$. 
analyze the morphology characteristics and elemental composition of soil samples, which were collected from eighteen regions in Shandong province, China. All samples exhibited their characteristic element contents, suggesting that it is possible to identify a specific soil sample from the sample pool by SEM-EDS analysis. This SEM-EDS- based analytical method will be helpful in the criminological analysis of trace soil samples.

\section{Materials and Methods}

2.1. Sample Collection. Eighteen surface soil samples were collected from different regions of Shandong Province, China. The samples were numbered from Sample 1 (S1) to Sample 18 (S18), corresponding to Zaozhuang, Yantai, Penglai (Yantai), Wulian (Rizhao), Changyi (Weifang), Rongcheng (Weihai), South mountainous area (Jinan), Liaocheng, Laiwu, Jiaozhou (Qingdao), Jiaxiang (Jining), Heze, Kenli (Dongying), Xiajin (Dezhou), Zouping (Bingzhou), Qufu, Taian, and Zibo, respectively.

2.2. SEM-EDS Analysis. All of the soil samples were dried for 72 hours in an oven at $110-120^{\circ} \mathrm{C}$ in order to remove moisture. Before they were subjected to the SEM-EDS analysis, samples were coated with a layer of gold using a goldplated machine for $120 \mathrm{~s}$. Sample analysis was performed in a Hitachi S-2500 Scanning Electron Microscope. The images were quantitatively analyzed with the software Image J [9]. SEM was equipped with an Oxford 3294X energy dispersive spectroscopic detector and was automated by Oxford Instruments INCA Energy and Feature Software. The results were detected and recorded with the Genesis 60E software.

\subsection{Results and Discussions}

2.3.1. SEM-EDS Analysis of the Samples. SEM is capable of imaging at a much higher resolution than light microscopes, owing to the small de Broglie wavelength of electrons. This property enables samples to be examined in fine details, as small as a single column of atoms, which is tens of thousands of times smaller than the smallest resolvable object under a light microscope. Objects can be observed in the limit of $0.1 \mathrm{~nm}\left(10^{-10} \mathrm{~m}\right)$. SEM images of the eighteen soil samples from different regions of Shandong Province were shown in Figure 1. All the soils are composed of micrometer-scale grains which consist of smaller particles with nanoscale structure characterization. The particle sizes of S1, S8, S10, and S13 were more homogeneous than those of others. Due to the presence of larger particles, S2, S5, and S6 were inhomogeneous, while S8 was the most homogeneous in all of the tested samples.

The particle area of these soil samples is shown in Figure 2. Samples from different regions showed varied diameters. For all of the samples, the area of soil particles was generally in the range of 100 to $500 \mu \mathrm{m}^{2}$. The increasing order of particle sizes was S11, S7, S18, S13, S12, S9, S16, S17, S15, S14, S4, S3, S2, S10, S1, S5, S6, and S8.

There are various elements in soil, such as oxygen, silicon, aluminum, iron, calcium, sodium, potassium, magnesium,

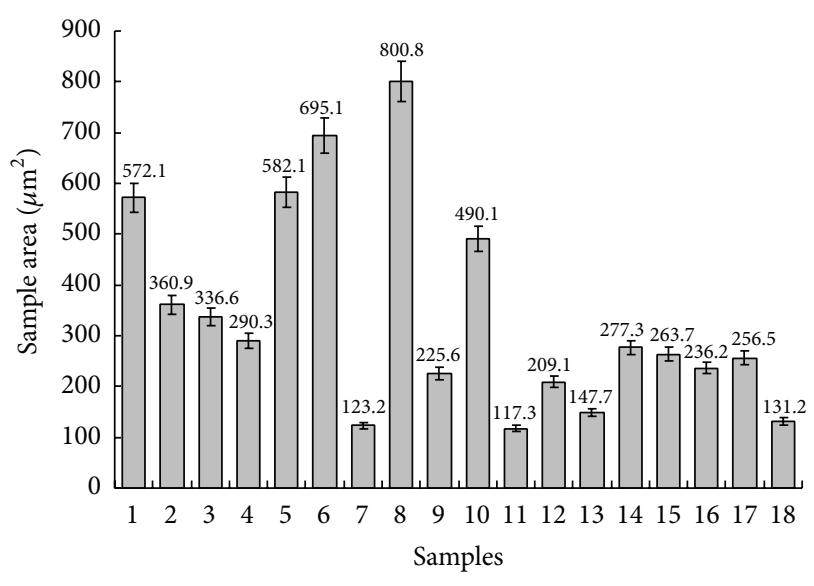

FIGURE 2: The particle area of soil samples from eighteen regions in Shandong province. Each bar represented the mean \pm SD of three replicates.

and titanium. Soil samples from different environments contained varied elements, which were characterized by element types and their content ratios.

The mass fraction and the atomic percentage of nine elements from the eighteen samples were measured by EDS. As shown in Figure 3, the contents of oxygen and silicon were the highest in all of the samples, because they are the composition elements of $\mathrm{SiO}_{2}$ and silicate, two of the most important components of soil. The contents of sodium and aluminium were relatively stable in all of these samples and ranged from $6.53 \%$ to $10.61 \%$ and from $0.46 \%$ to $1.54 \%$, respectively. Contents of other elements varied obviously among different samples. Contents of calcium and magnesium in S11, S12, and S13 were higher than other samples, because many lands in Kenli, Xiajin, and Zouping are saline and alkaline. The content of the element titanium was the highest in S3 in comparison with all of the other samples, and the contents of the elements iron and potassium in S3 were the lowest, because this sample was collected near a coating plant. S9, S12, and S18 contained the lowest amount of magnesium, $0.98 \%$, $0.80 \%$, and $0.87 \%$, respectively. The contents of the element calcium in the soil samples S1, S6, and S7 were higher than those in other samples, likely due to the native limestone constituents.

The atomic percentages of oxygen, silicon, sodium, and aluminium were relatively stable in all of these samples. Contents of calcium and magnesium were higher in S11, S12, and S13 than other samples. The atomic percentage of nine elements (Figure 4) showed the same trend as the mass fraction in Figure 3.

\section{Conclusion}

SEM-EDS-based analytical method could be used for the automatic examination and measurement of the geometric and morphological parameters, including the homogeneities and particle sizes of soil samples, which are important for the identification of the soil. The difference in soil elemental 


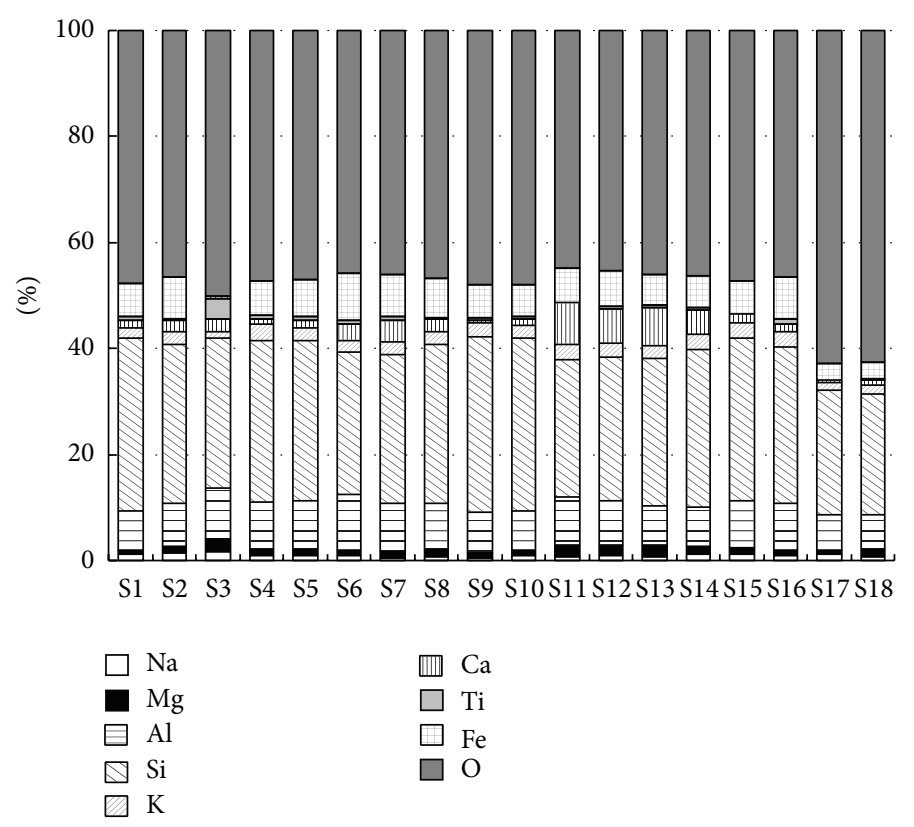

FIGURE 3: Element mass fraction of soil samples from eighteen regions in Shandong province.

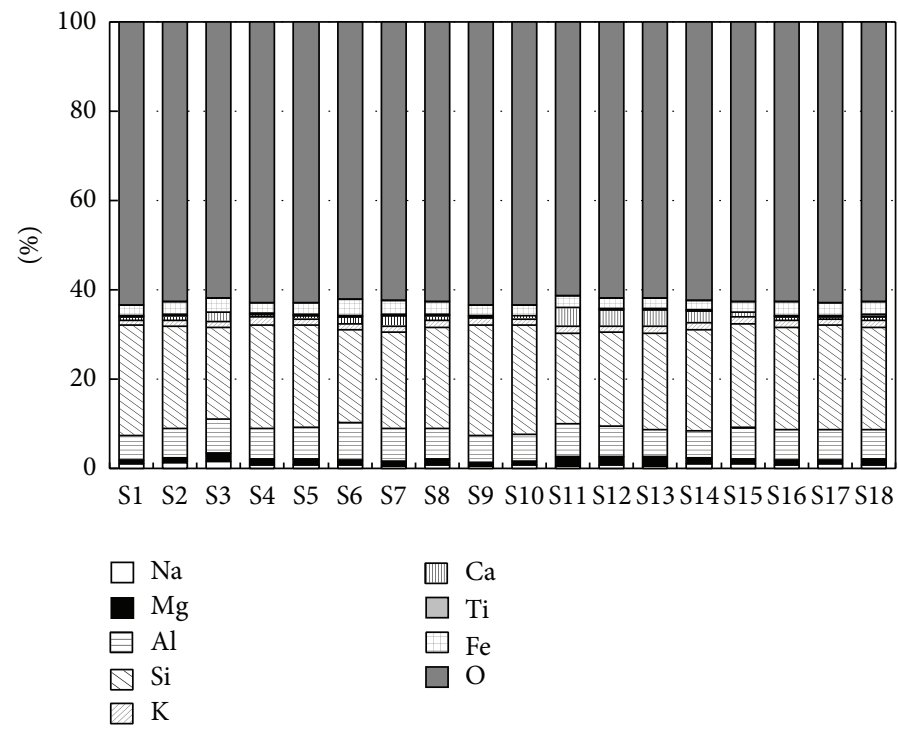

FIgURE 4: Element atomic percentage of soil from eighteen regions in Shandong Province.

components represents the variety of the soil texture from different regions, which is important when narrowing the tracking range of investigation. As a soil database in China is still in its infancy, this research will be helpful in performing the convenient batch detections of a large number of soil samples to establish a national and global SEM-EDS database for forensic science.

\section{Conflict of Interests}

The authors declare that there is no conflict of interests regarding the publication of this paper.

\section{Authors' Contribution}

Yingzi Liu and Yumei Li contributed equally to this work.

\section{Acknowledgments}

This work was supported by the National Natural Science Foundation of China (Project nos. 31100088 and 31300045), Shandong province science and technology development plan (Grant nos. 2013GSF12006 and 2012GGB01172), Foundation of University of Jinan (XKY1324), and Xinjiang Production \& Construction Corps Key Laboratory of Protection 
and Utilization of Biological Resources in Tarim Basin (Grant no. BRYB1405).

\section{References}

[1] Y. Ge, L. Hu, and S. Chen, "Application and examination methods of soil for forensic science," Journal of Chinese Legal Medical Science, vol. 19, no. 4, pp. 260-261, 2004.

[2] S. A. Larson, Developing a High Throughput Protocol for Using Soil Molecular Biology as Trace Evidence, University of Nebraska-Lincoln, Lincoln, Neb, USA, 2012.

[3] B. D. Lee, T. N. Williamson, and R. C. Graham, "Identification of stolen rare palm trees by soil morphological and mineralogical properties," Journal of Forensic Sciences, vol. 47, no. 1, pp. 190194, 2002.

[4] M. Horrocks and K. J. Walsh, "Pollen on grass clippings: putting the suspect at the scene of the crime," Journal of Forensic Sciences, vol. 46, no. 4, pp. 947-949, 2001.

[5] L. A. Daugherty, "Soil science contribution to an airplane crash investigation, Ruidoso, New Mexico," Journal of Forensic Sciences, vol. 42, no. 3, pp. 401-405, 1997.

[6] N. Petraco and T. Kubic, "A density gradient technique for use in forensic soil analysis," Journal of Forensic Sciences, vol. 45, no. 4, pp. 872-873, 2000.

[7] S. Cengiz, A. C. Karaca, I. Çakir, H. B. Üner, and A. Sevindik, "SEM-EDS analysis and discrimination of forensic soil," Forensic Science International, vol. 141, no. 1, pp. 33-37, 2004.

[8] J. Wang, Y. Li, Z. Zhao et al., "T-RFLP analysis of soil microbial community from shandong province for forensic science," Advanced Materials Research, vol. 599, pp. 250-253, 2012.

[9] http://rsb.info.nih.gov/. 

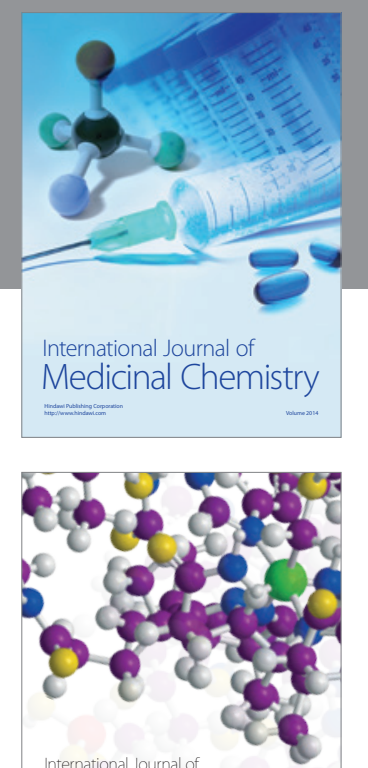

\section{Carbohydrate} Chemistry

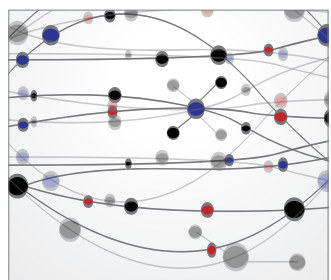

The Scientific World Journal
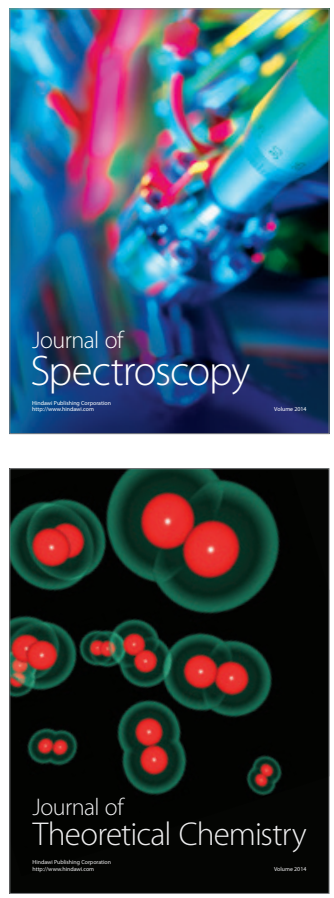
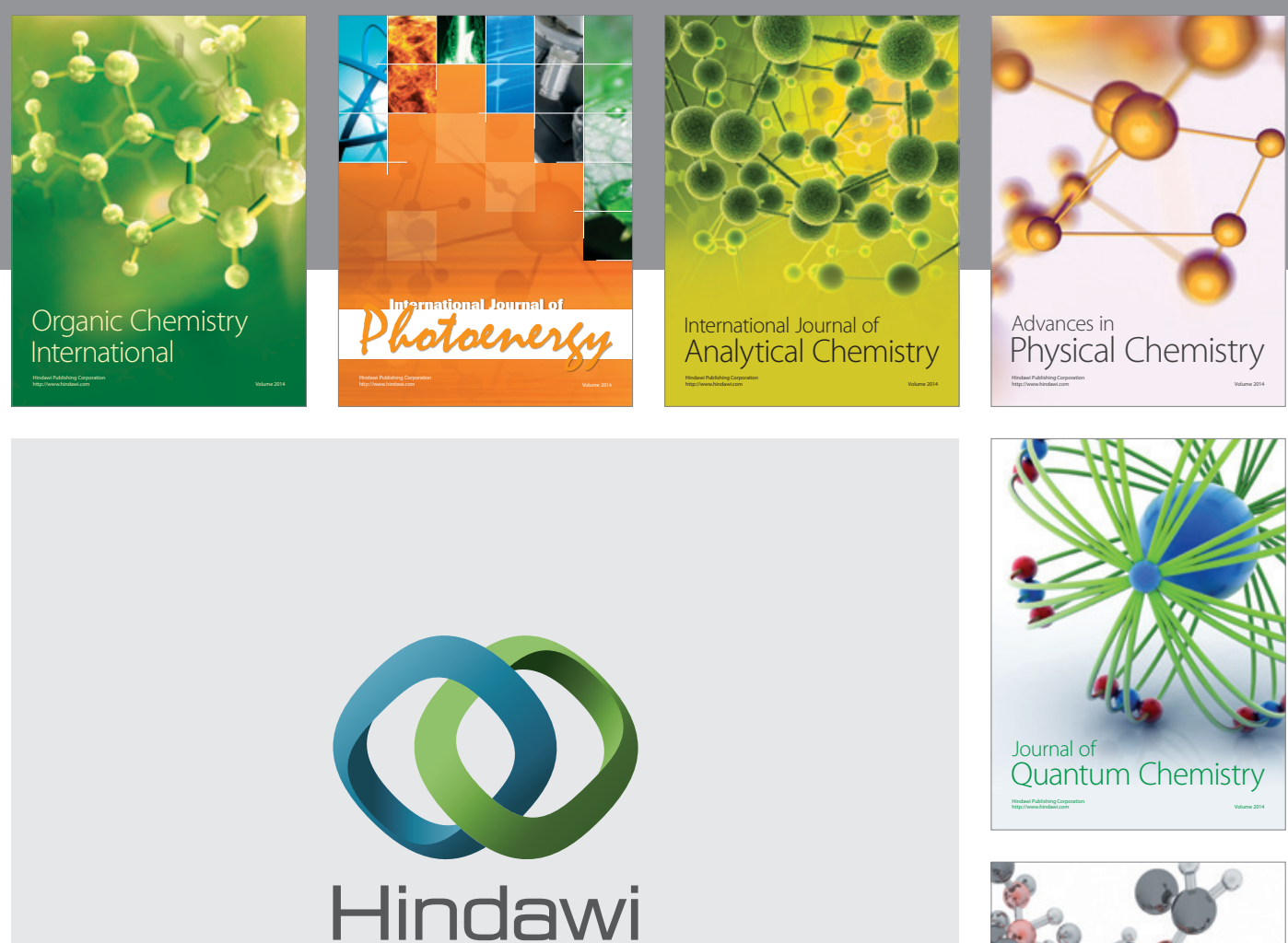

Submit your manuscripts at

http://www.hindawi.com

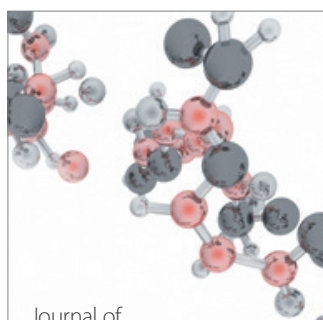

Analytical Methods

in Chemistry

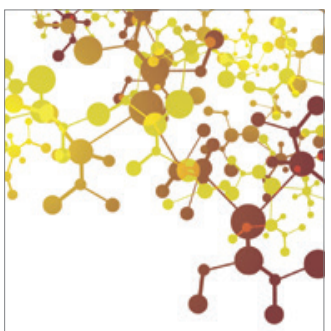

Journal of

Applied Chemistry

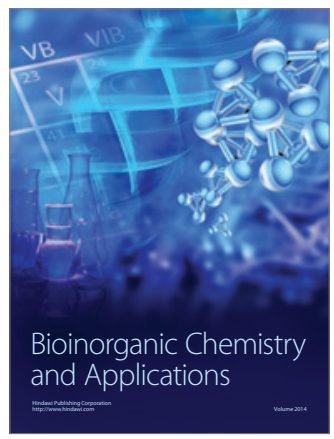

Inorganic Chemistry
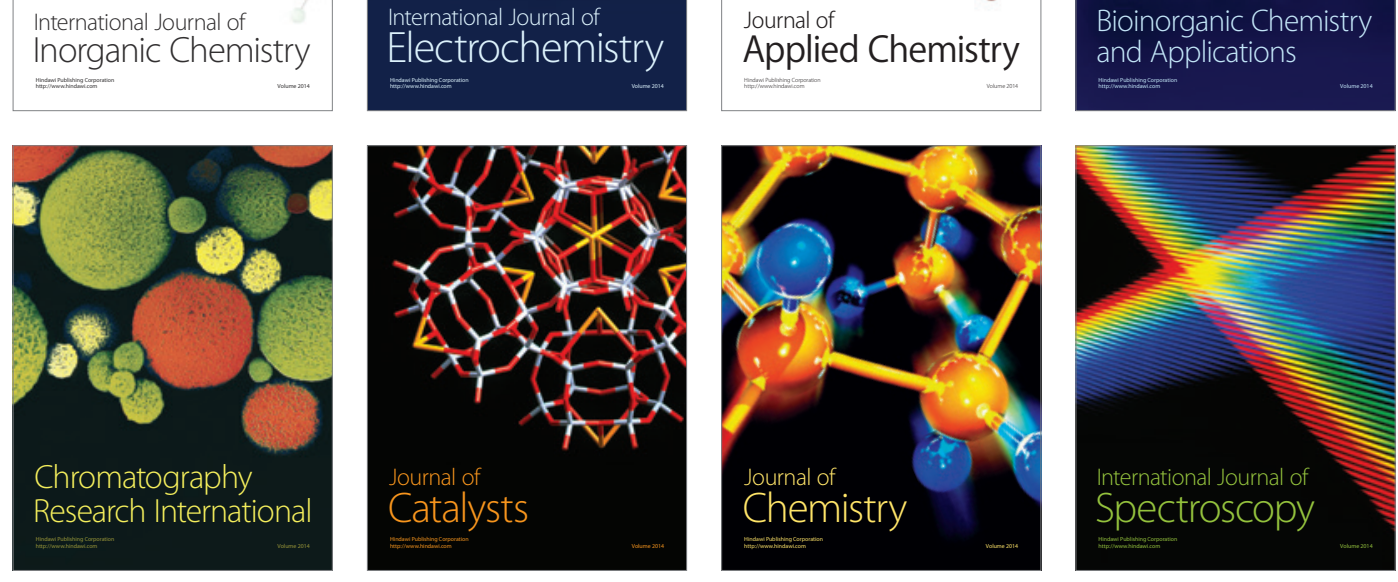